\title{
Immunological effects of adjuvants in subsets of antigen presenting cells of cancer patients undergoing chemotherapy
}

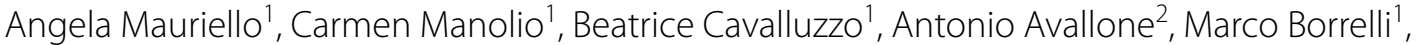 \\ Alessandro Morabito ${ }^{3}$, Emanuele lovine ${ }^{3}$, Angela Chambery ${ }^{4}$, Rosita Russo ${ }^{4}$, Maria Lina Tornesello ${ }^{5}$, \\ Franco M. Buonaguro ${ }^{5}$, Maria Tagliamonte ${ }^{1 *}$ and Luigi Buonaguro ${ }^{1 *}$ (D)
}

\begin{abstract}
Background: We have previously shown that HCC patients and healthy subjects are equally responsive to a RNAdjuvant ${ }^{\circledR}$, a novel TLR-7/8/RIG-I agonist based on noncoding RNA developed by CureVac, by an ex vivo evaluation. However, the immunological effect of adjuvants on immune cells from cancer patients undergoing chemotherapy remains to be demonstrated. Different adjuvants currently used in cancer vaccine clinical trials were evaluated in the present study on immune cells from cancer patients before and after chemotherapy in an ex vivo setting.

Methods: PBMCs were obtained from 4 healthy volunteers and 23 patients affected by either colon (OMA) or lung cancer (OT). The effect of $\mathrm{CpG}$, Poly I:C, Imiquimod and RNA-based adjuvant (RNAdjuvant ${ }^{\circledR}$ ) was assessed using a multiparametric approach to analyze network dynamics of early immune responses. Evaluation of CD80, CD86 and HLA-DR expression as well as the downstream effect on $\mathrm{CD}^{+}{ }^{+} \mathrm{T}$ cell phenotyping was performed by flow cytometry; cytokine and chemokine production was evaluated by Bio-Plex ProTM.

Results: Treatment with RNAdjuvant ${ }^{\circledR}$ induced the strongest response in cancer patients in terms of activation of innate and adoptive immunity. Indeed, CD80, CD86 and HLA-DR expression was found upregulated in circulating dendritic cells, which promoted a CD4 ${ }^{+} T$ cell differentiation towards an effector phenotype. RNAdjuvant ${ }^{\circledR}$ was the only one to induce most of the cytokines/chemokines tested with a pronounced Th1 cytokine pattern. According to the different parameters evaluated in the study, no clear cut difference in immune response to adjuvants was observed between healthy subjects and cancer patients. Moreover, in the latter group, the chemotherapy treatment did not consistently correlate to a significant altered response in the different parameters.

Conclusions: The present study is the first analysis of immunological effects induced by adjuvants in cancer patients who undergo chemotherapy, who are enrolled in the currently ongoing cancer vaccine clinical trials. The results show that the RNAdjuvant ${ }^{\circledR}$ is a potent and Th1 driving adjuvant, compared to those tested in the present study. Most importantly, it is demonstrated that chemotherapy does not significantly impair the immune system, implying that cancer patients are likely to respond to a cancer vaccine even after a chemotherapy treatment.
\end{abstract}

\footnotetext{
*Correspondence: m.tagliamonte@istitutotumori.na.it;

I.buonaguro@istitutotumori.na.it

${ }^{1}$ Laboratory of Cancer Immunoregulation, Istituto Nazionale per lo Studio

e la Cura dei Tumori, IRCCS "Fondazione Pascale", Via Mariano Semmola,

80131 Naples, Italy

Full list of author information is available at the end of the article
}

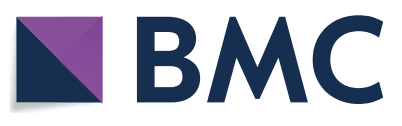

(c) The Author(s) 2020. This article is licensed under a Creative Commons Attribution 4.0 International License, which permits use, sharing, adaptation, distribution and reproduction in any medium or format, as long as you give appropriate credit to the original author(s) and the source, provide a link to the Creative Commons licence, and indicate if changes were made. The images or other third party material in this article are included in the article's Creative Commons licence, unless indicated otherwise in a credit line to the material. If material is not included in the article's Creative Commons licence and your intended use is not permitted by statutory regulation or exceeds the permitted use, you will need to obtain permission directly from the copyright holder. To view a copy of this licence, visit http://creativeco mmons.org/licenses/by/4.0/. The Creative Commons Public Domain Dedication waiver (http://creativecommons.org/publicdomain/ zero/1.0/) applies to the data made available in this article, unless otherwise stated in a credit line to the data. 
Keywords: Adjuvant, Colon cancer, Lung cancer, Immune-response, Cancer vaccine

\section{Background}

Cancer immunotherapy is a recently fast growing field and several therapeutic cancer vaccine strategies are being investigated [1]. However, the efficacy of such strategies is often limited, due to the low immunogenicity of administered antigens which requires to be potentiated by a strong adjuvant [2-4]. Although the field is continuously evolving, only a few number of adjuvants have been approved for human use to date [5].

The development of new adjuvants has been guided in the last years by the detailed comprehension of the central role played by the innate immunity to initiate and direct the adaptive immune response. In particular, the pattern recognition receptors (PRRs) in cells of the innate immune system act as sensors for bacterial and viral nucleic acids (pathogen-associated molecular patterns; PAMPs) and induce the innate immune response by activation of a signaling cascade resulting in the upregulation of inflammatory cytokines, chemokines, and type I IFNs. This will eventually end up in triggering a robust adaptive immune response [6]. Therefore, PRRs represent the ideal target for vaccine adjuvants to initiate and boost an antigen-specific $\mathrm{T}$ cell response (reviewed in [7]).

In this framework, several TLR ligands have been extensively evaluated as vaccine adjuvants in pre-clinical as well as human clinical setting (reviewed in [8]). In particular, the polyinosinic-polycytidylic acid (Poly I:C) is a synthetic TLR-3 agonist reported as type 1 adjuvant able to elicit antigen-specific CTL, antibody and Th1 type immune responses when included in cancer vaccines [9-11]. Synthetic CpG oligodeoxynucleotides (ODNs) are TLR-9 agonists able to induce type I IFNs as well as proinflammatory cytokine production, generating Th1 type cellular and humoral immune responses [12, 13]. Recently, the CpG-ODN was approved for the first time for application in humans, and Heplisav- $B$, a hepatitis $\mathrm{B}$ vaccine containing $\mathrm{CpG-ODN}$ as an adjuvant, was approved by the United States Food and Drug Administration [14]. Imiquimod, is a synthetic TLR7/8 agonist, reported to induce an adjuvant activity able to boost antigen-specific humoral and Th1 type cellular immune responses $[15,16]$. However, systemic toxicity has been reported and the only TLR7 agonist-based drug approved for clinical use as an anti-tumor agent is the topically applied Aldara cream for patients with precancerous skin lesions [17]. Nevertheless, the field is still open for new adjuvants and in particular for molecules able to elicit a balanced humoral and cellular immune response for potential application in both preventive and therapeutic vaccines.

In this framework, RNAdjuvant ${ }^{\circledR}$, a TLR 7/8/RIG I agonist based on noncoding RNA has been shown to induce a potent Th1 and long-lasting immune response if used as adjuvant for peptide vaccines in preclinical models, including cancer tumor models $[18,19]$.

Therapeutic cancer vaccines are designed to be administered in patients already affected by a disease such as cancer which is considered to induce an immune compromised situation, especially as consequence of standard-of-care chemotherapy. Indeed, the cytostastic effect is not specific to cancer cells and may affect all replicating cells, including immune cells, possibly resulting in the impairment of effector $\mathrm{T}$ cells and activation of immunosuppressive mechanisms [20]. However, the lymphodepletion caused by chemotherapy can turn into a reset of the immune system as consequence of the rebound replenishment of immune cells [21], with the emergence of competent effector cells also with anticancer activity [22]. Indeed, cancer patients have been shown to respond to influenza virus vaccination during chemotherapy [23, 24] and specific anti-infective vaccination guidelines for patients with hematological malignancies have been designed [25].

These observations indicate that routine chemotherapy is compatible with the initiation of an immune response and anticancer vaccines have been shown to elicit antitumor immune responses in patients during chemotherapy (reviewed in [26]).

Adjuvants are a key component of vaccine formulations, historically used as preventive strategy in healthy subjects. Provided all the immunological implications related to the cancer disease and the chemotherapy, an experimental validation is needed to prove that adjuvants are equally effective in healthy subjects and cancer patients. For such a reason, taking advantage of an ex vivo multiparametric platform, developed and fully validated by our group [27-33], we have previously shown that HCC patients who do not undergo chemotherapy and healthy subjects are equally responsive to the RNAdjuvant ${ }^{\circledR}$ [34]. However, the immunological effect of adjuvants in cancer patients undergoing chemotherapy remains to be demonstrated.

To this aim, adjuvants currently used in cancer vaccine clinical trials were evaluated in an ex vivo setting on immune cells from patients affected by colon cancer and lung cancer before and after chemotherapy. 
Among the adjuvants evaluated in the study, the RNAdjuvant ${ }^{\circledR}$ elicited the strongest response in cancer patients in terms of activation of innate and adoptive immunity. It was the only one to induce production of IFN $\gamma$ Th1 cytokine and a potent inducer of both proinflammatory cytokines and chemokines. Very importantly, immune response induced in cancer patients was significant although of lower potency compared to healthy subjects. Nevertheless, the narrow differences between pre and post-chemotherapy samples indicates that cancer patients may well respond to therapeutic cancer vaccines even after chemotherapy.

\section{Materials and methods}

\section{Enrolled subjects}

Peripheral blood was obtained by venipuncture from 4 healthy volunteers, eleven colon cancer (OMA) and twelve lung cancer patients (OT) before and after chemotherapy. Post-chemotherapy blood samples were obtained about 3 weeks after the end of therapy (median 25 days). All human specimens were obtained and processed at the National Cancer Institute in Naples under informed consent, as approved by the Institutional Review Board.

\section{PBMC isolation}

Fresh human PBMCs were isolated by Ficoll-Hypaque density gradient centrifugation and plated in six-well plates at a concentration of approximately $1 \times 10^{6}$ cells/ well in a maximum volume of $2 \mathrm{ml} /$ well. Isolated PBMCs were incubated for $24 \mathrm{~h}$ (short-term culture) or for 7 days (medium-term culture) in RPMI 1640 medium.

\section{Cell culture medium}

PBMCs culture medium consisted of RPMI 1640 medium (Life Technologies, Carlsbad, CA) supplemented with $2 \mathrm{mM} \mathrm{L-glutamine} \mathrm{(Sigma),} \mathrm{10 \%} \mathrm{fetal} \mathrm{calf}$ serum (Life Technologies) and 2\% penicillin/streptomycin $(5000$ I.U. $/ 5 \mathrm{mg} / \mathrm{ml}$, MP Biomedicals). Recombinant interleukin-2 (rIL-2; R\&D Systems, Minneapolis, Minn.) was added at a concentration of $75 \mathrm{U} / \mathrm{ml}$ for mediumterm culture (every 2 days).

\section{Cell treatment}

PBMCs were incubated for $24 \mathrm{~h}$ (short-term culture) with the different adjuvants: $20 \mu \mathrm{g} / \mathrm{ml}$ of RNAdjuvant ${ }^{\circledR}$ provided by CureVac (Tübingen, Germany), $6 \mu \mathrm{g} / \mathrm{ml}$ of Poly I:C, $5 \mu \mathrm{g} / \mathrm{ml}$ of Imiquimod, $2,5 \mu \mathrm{g} / \mathrm{ml}$ of CpG ODN 2006, $2 \mu \mathrm{g} / \mathrm{ml}$ of lipopolysaccharide (LPS) as positive control (from Salmonella enterica serotype Minnesota, purity $>99.0 \%$ ). Alternatively, PBMCs were incubated for 7 days (medium-term culture) with the same concentration of RNAdjuvant ${ }^{\circledR}$ or LPS, plus IL-2 added at day 0,2 and 4. PBS was used as negative control. At the end of the

\begin{tabular}{llll}
$\begin{array}{l}\text { Table } 1 \text { Informations } \\
\text { of enrolled patients }\end{array}$ & and & chemotherapy treatment \\
\hline Samples & Sex & Age & Treatment \\
\hline Healthy 1 & M & 50 & \\
Healthy 2 & F & 40 & \\
Healthy 3 & M & 28 & \\
Healthy 4 & F & 23 & \\
OT 1 & M & 72 & Cisplatin + vinorelbine \\
OT 2 & F & 73 & Gemcitabine \\
OT 3 & M & 72 & Carboplatin + gemcitabine \\
OT 4 & F & 68 & Cisplatin + etoposide \\
OT 5 & F & 81 & Carboplatin + alimta \\
OT 6 & M & 61 & Cisplatin + gemcitabine \\
OT 7 & M & 62 & Carboplatin + gemcitabine \\
OT 8 & F & 69 & Cisplatin + gemcitabine \\
OT 9 & M & 72 & Carboplatin + vinorelbine \\
OT 10 & M & 78 & Cisplatin + gemcitabine \\
OT 11 & F & 64 & Carboplatin + pemetrexed \\
OT 12 & F & 62 & Cisplatin + pemetrexed \\
OMA 1 & F & 61 & XELOX \\
OMA 2 & F & 54 & XELOX \\
OMA 3 & M & 56 & XELOX \\
OMA 4 & M & 62 & XELOX \\
OMA 5 & M & 52 & XELOX \\
OMA 6 & F & 60 & XELOX \\
OMA 7 & M & 62 & XELOX \\
OMA 8 & M & 60 & XELOX \\
OMA 9 & M & 66 & XELOX \\
OMA 10 & M & 55 & XELOX \\
OMA 11 & M & 66 & XELOX \\
\hline & & & \\
\hline
\end{tabular}

incubation, PBMCs were harvested, washed with PBS $1 \times$ $(137 \mathrm{mM} \mathrm{NaCl}, 2.7 \mathrm{mM} \mathrm{KCl}, 10 \mathrm{mM} \mathrm{Na} 2 \mathrm{HPO}, 2 \mathrm{mM}$ $\mathrm{KH}_{2} \mathrm{PO}_{4}, \mathrm{pH}$ 7.2) without Calcium and Magnesium and analyzed by flow cytometry. All cell supernatants were collected for quantification of cytokine and chemokine production.

\section{Flow cytometry}

Short-term culture PBMCs were incubated for $30 \mathrm{~min}$ at $4{ }^{\circ} \mathrm{C}$ with human monoclonal antibodies specific for CD80, CD86, HLA-DR, CD123, CD11c and CD14 (BD Pharmingen, San Diego, $C A$ ), washed and then analyzed with the Attune NxT flow cytometer (Life Technology). In particular, monocytes, $\mathrm{mDCs}$ and $\mathrm{pDCs}$ were identified according to the following gating strategy. The monocyte fraction was selected from the whole PBMCs and, within such a fraction, cells were selected according to the positivity for CD14 marker $\left(\mathrm{CD} 14^{+}\right.$: monocytes; CD14 ${ }^{-}$: dendritic cells). CD14 ${ }^{-}$DCs were further divided 


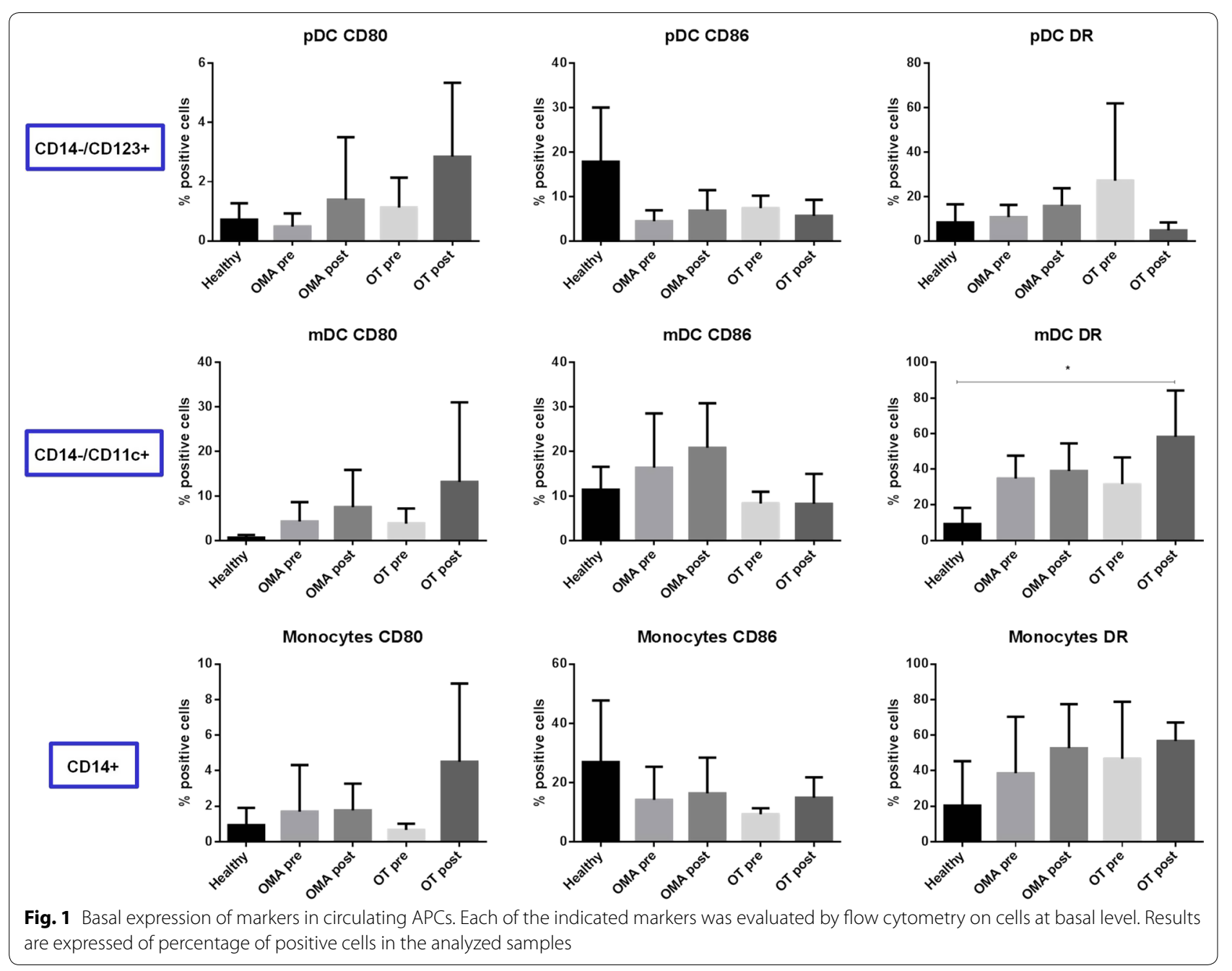

in $\mathrm{mDCs}$, if positive for CD11c, and $\mathrm{pDCs}$, if positive for CD123. On cell subtypes defined in this way, the percentage of CD80, CD86 and HLA-DR positivity was assessed.

\section{T-maturation analysis}

Medium-term culture PBMCs were analyzed for markers of $\mathrm{CD}^{+}{ }^{+}$Tcell phenotyping by flow cytometry. PBMCs were collected, washed in PBS, centrifuged and incubated for $30 \mathrm{~min}$. at $4{ }^{\circ} \mathrm{C}$ with monoclonal antibodies specific for the surface molecules of human $\mathrm{T}$ lymphocytes: CD4, CD45RA, CD45RO, CD62L (BD Pharmingen, San Diego, CA). Naïve (TN) $\mathrm{CD}^{+}{ }^{+} \mathrm{T}$ were identified as $\mathrm{CD}^{+} / \mathrm{CD} 4 \mathrm{RA}^{+} / \mathrm{CD} 2 \mathrm{~L}^{+}$; effector cells (TE) as $\mathrm{CD} 4^{+} /$ CD45RA ${ }^{+} / \mathrm{CD} 6 \mathrm{~L}^{-}$; central memory (TCM) as $\mathrm{CD} 4^{+} /$ $\mathrm{CD} 45 \mathrm{RO}^{+} / \mathrm{CD} 6 \mathrm{~L}^{+}$; effector memory $(\mathrm{TEM})$ as $\mathrm{CD}^{+} /$ $\mathrm{CD}^{2} 5 \mathrm{RO}^{+} / \mathrm{CD}^{2} 2 \mathrm{~L}^{-}$.

\section{Cytokine analysis}

At the time of cell harvest, supernatants were also collected and stored at $-80{ }^{\circ} \mathrm{C}$ until use. Cytokine and chemokine production was evaluated by using a BioPlex MAGPIX Multiplex Reader system (Bio-Rad, Milan, Italy) equipped with a Bio-Plex Manager software $\mathrm{v}$ 6.1 (BioRad) according to manufacturer's instructions [35]. All washing steps were performed on the Bio-Plex magnetic wash station (BioRad). Measurements were performed in triplicate on samples $(50 \mu \mathrm{l})$ using the BioPlex Pro Human Cytokine 40-plex assay kit (Cat. No. 171AK99MR2, BioRad). Standard curves optimization and the calculation of analyte concentrations were performed by using the Bio-Plex Manager software. Data were expressed as mean $\pm \mathrm{SD}$.

\section{Statistical analysis}

Statistical analysis was performed using Graphpad Prism 6 software (Graphpad Software, La Jolla, CA, USA) and the results of ANOVA nonparametric test 


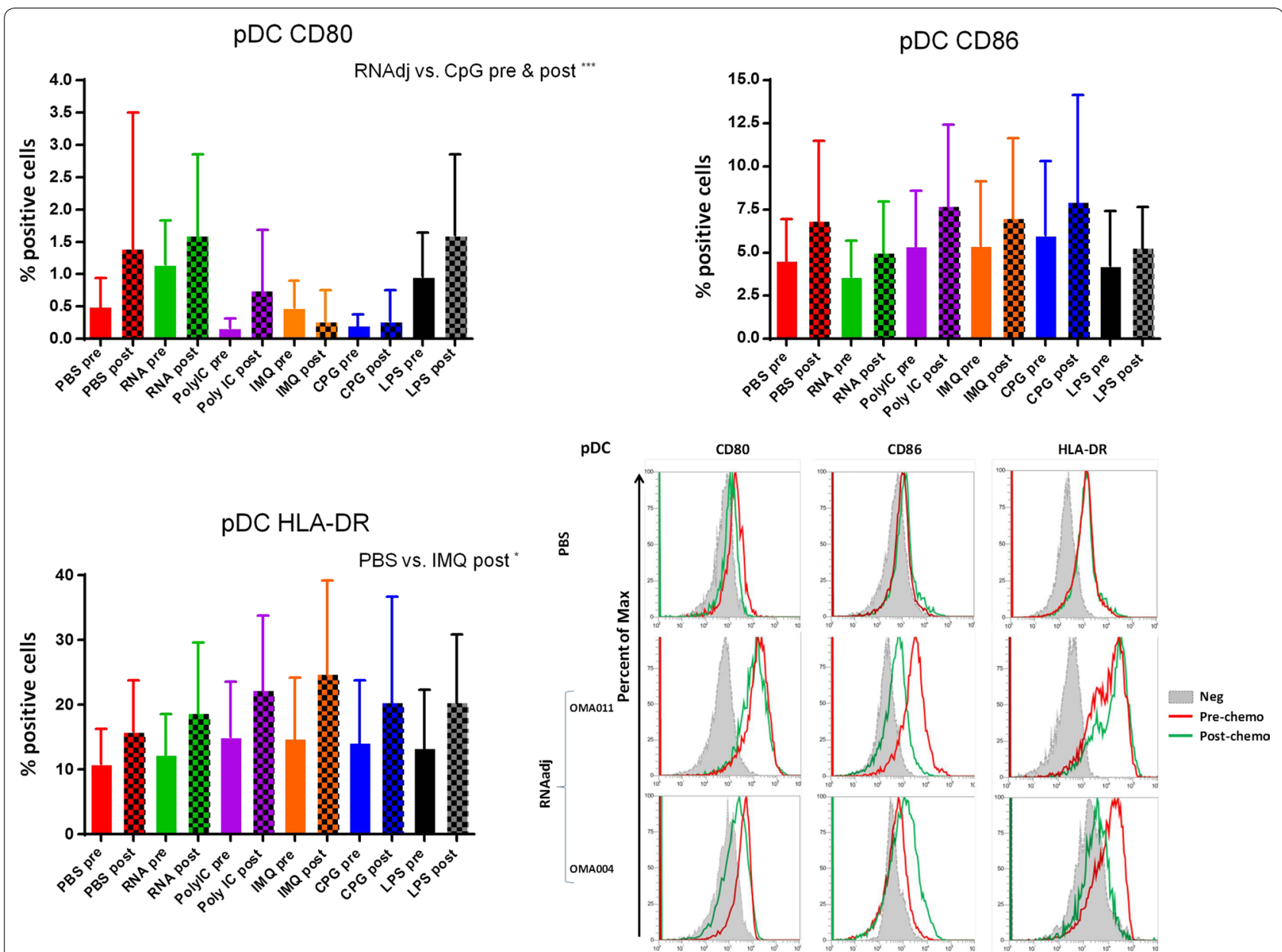

Fig. 2 Expression of markers induced by adjuvants in circulating pDCs from colon ca patients. Each of the indicated markers was evaluated by flow cytometry on cells after ex vivo treatment with adjuvants. Samples from colon cancer patients were collected pre and post-chemotherapy. Results are expressed of percentage of positive cells in the analyzed samples. Expression of individual markers induced by RNAdjuvant ${ }^{\circledR}$ in two individual patients is shown as plot

were considered statistically significant at a level of $\mathrm{p}<0.05$. Normally distributed data were represented as mean \pm S.E.M.

\section{Results}

\section{Clinical parameters of subjects included in the analysis}

Twenty-three subjects were enrolled in the present study. Eleven subjects were affected by colon cancer (OMA); twelve subjects were affected by non-small cell lung carcinoma (OT). Fourteen were males and nine were females with a median age of 65 (range 52-81). Four healthy donors were enrolled as controls. Clinical parameters and chemotherapy treatment of enrolled subjects are described in Table 1. Due to technical reasons, only three samples from OT patients were included in the present analysis.
Basal expression of maturation markers in circulating APCs The expression of CD80, CD86 and HLA-DR molecules was examined in circulating antigen-presenting cells (APCs) by flow cytometry. In particular, their expression on $\mathrm{CD} 14^{+}$monocytes, $\mathrm{CD} 14^{-} \mathrm{CD} 11 \mathrm{c}^{+}$myeloid dendritic cells (mDCs) and $\mathrm{CD} 14^{-} \mathrm{CD} 123^{+}$plasmacytoid dendritic cells (pDCs) were evaluated in parallel [34].

The basal expression of the CD80, CD86 and HLADR molecules in the three APC populations was largely comparable between the healthy subjects and the cancer patients, before and after chemotherapy (Fig. 1). In particular, CD80 and HLA-DR were very low in healthy subjects and showed a trend of increased expression in cancer patients, especially after chemotherapy, with different degree in the three cell populations. Such an increased expression reached the statistical significance only for HLA-DR in monocytes from OT patients after chemotherapy. On the contrary, CD86 expression was 

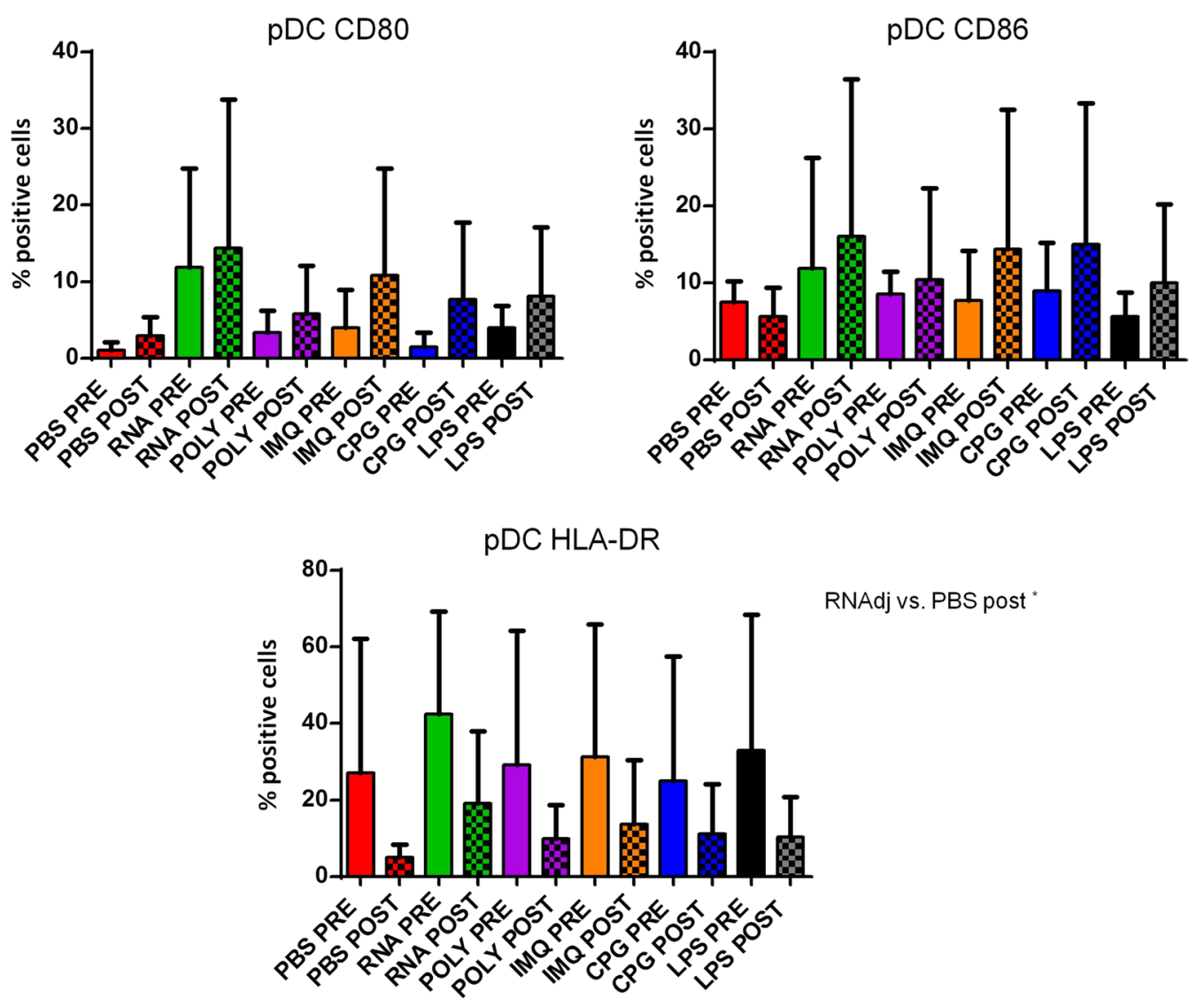

Fig. 3 Expression of markers induced by adjuvants in circulating pDCs from lung ca patients. Each of the indicated markers was evaluated by flow cytometry on cells after ex vivo treatment with adjuvants. Samples from lung cancer patients were collected pre and post-chemotherapy. Results are expressed of percentage of positive cells in the analyzed samples

high in healthy subjects and showed limited variation in cancer patients, without reaching a statistical significance (Fig. 1).

\section{Induction of maturation markers in plasmacytoid dendritic} cells by different adjuvants

The expression of CD80, CD86 and HLA-DR molecules was evaluated in $\mathrm{CD} 14^{-} \mathrm{CD} 123^{+}$plasmacytoid dendritic cells (pDCs) from cancer patients, before and after chemotherapy, upon ex vivo treatment with the adjuvants. The dose used for each adjuvant was selected according to recommendations provided by producers and reported in the literature [19, 34, 36-38]. Results in OMA patients showed that none of the adjuvants used in this study was able to induce a statistical significant increased expression in pDCs of the maturation markers compared to the negative control. The only exception was the IMQ that induced an upregulation of HLA-DR molecules in pDCs after the chemotherapy (Fig. 2; Additional file 1: Figure S1). Patients showed a great variable responsiveness to adjuvants and an example of low and high responders is shown in Fig. 2. Similar results were observed in pDCs derived from OT patients. In this setting, the RNAdjuvant ${ }^{\circledR}$ was the only ex vivo treatment to induce a trend of increased expression of CD80 and HLA-DR compared to the negative control, in pre and post-treatment samples respectively (Fig. 3; Additional file 1: Figure S2). In healthy subjects the effects of adjuvants were evident and statistically significant. In particular, the expression of the three activation markers was increased by the adjuvants compared to negative control, with individual specificity. The comparison between the different adjuvants showed unique features in the induction of individual activation markers (Fig. 4).

\section{Induction of maturation markers in myeloid dendritic cells by different adjuvants}

The same kind of evaluation was performed on $\mathrm{CD} 14^{-} \mathrm{CD} 11 \mathrm{c}^{+}$myeloid dendritic cells (mDCs). Results in OMA patients showed that only the RNAdjuvant ${ }^{\circledR}$ 

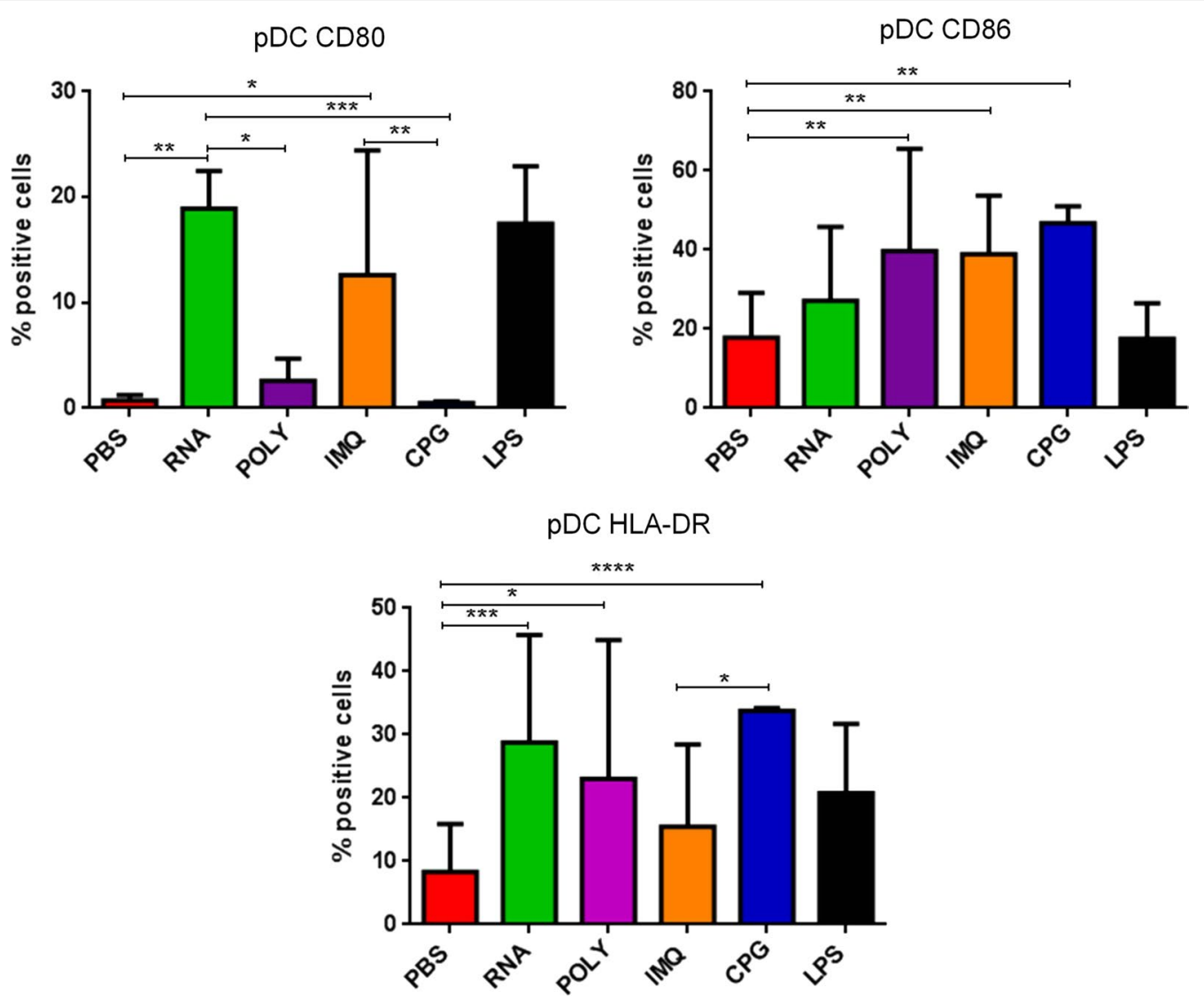

Fig. 4 Expression of markers induced by adjuvants in circulating pDCs from healthy subjects. Each of the indicated markers was evaluated by flow cytometry on cells after ex vivo treatment with adjuvants. Results are expressed of percentage of positive cells in the analyzed samples

and $\mathrm{CpG}$ were able to induce in $\mathrm{mDCs}$ a statistical significant increased expression of the CD80 and CD86 maturation markers in samples pre-chemotherapy compared to the negative control, respectively. Moreover, the RNAdjuvant ${ }^{\circledR}$ induced a statistically significant increase of the CD80 molecule in mDCs compared to Poly I:C in pre-treatment samples and CpG in both pre and post-treatment samples. On the contrary, $\mathrm{CpG}$ and Poly I:C induced a statistically significant increase of the CD86 molecule in mDCs compared to RNAdjuvant ${ }^{\circledR}$ in both pre and post-chemotherapy samples. No significant difference in expression was observed for the HLA-DR (Fig. 5; Additional file 1: Figure S3). Also for mDCs, patients showed a great variable responsiveness to adjuvants and an example of low and high responders is shown in Fig. 5. Results obtained in $\mathrm{mDC}$ derived from OT patients showed a trend to increased expression of the maturation markers compared to the negative control induced by most of the adjuvants analyzed in the study, without reaching a statistical significance. No significant difference was observed between the effects induced by the different adjuvants, with the exception of the CD80, whose expression was significantly induced by the RNAdjuvant ${ }^{\circledR}$ compared to the $\mathrm{CpG}$ in post-chemotherapy samples (Fig. 6; Additional file 1: Figure S4). In healthy subjects the effects of adjuvants were evident and statistically significant only for the CD80 and CD86. In particular, the RNAdjuvant ${ }^{\circledR}$ increased the expression of both activation markers compared to negative control. Moreover, the RNAdjuvant ${ }^{\circledR}$ induced the strongest expression of the CD80 compared to all other adjuvants. Finally, the Poly I:C increased the expression of the CD86 marker compared to the negative control (Fig. 7). 


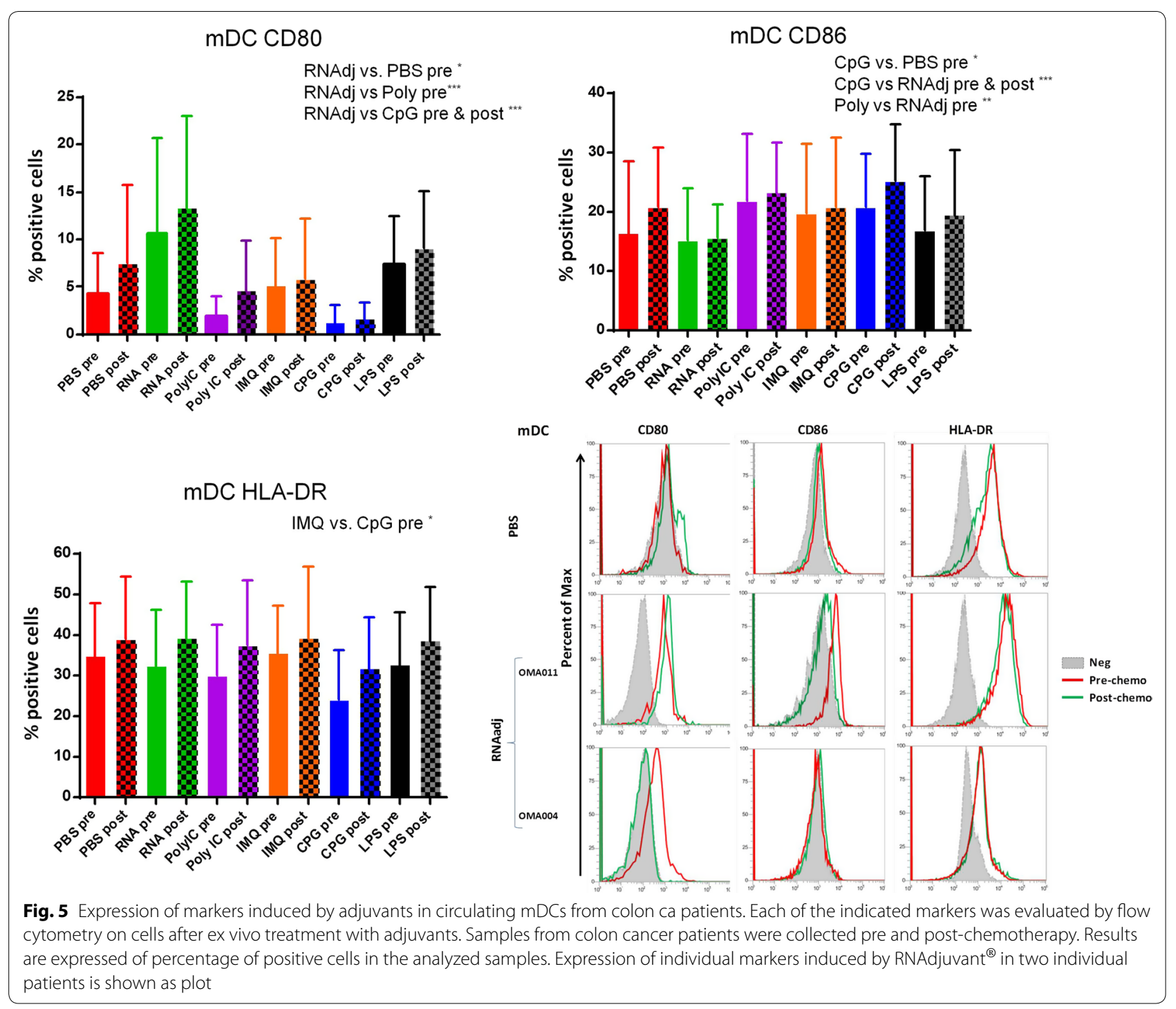

\section{Induction of maturation markers in monocytes by different} adjuvants

Finally, the expression of CD80, CD86 and HLA-DR molecules was evaluated in $\mathrm{CD}_{1} 4^{+}$monocytes. Results in OMA patients showed that only the RNAdjuvant ${ }^{\circledR}$ induced a statistical significant increased expression of the CD80 compared to the negative control in samples pre and post-chemotherapy. Moreover, the RNAdjuvant ${ }^{\circledR}$ induced a statistically significant increase of the CD80 molecule compared to all other adjuvants in pre and post-chemotherapy samples. The expression of CD86 was not significantly increased by adjuvants compared to the negative control, with the exception of Poly I:C in pre-chemotherapy samples. Similar results were observed for HLA-DR, with the exception of a surprising significant down regulation induced by RNAdjuvant ${ }^{\circledR}$ (Fig. 8; Additional file 1: Figure S5). An example of low and high responders to RNAdjuvant ${ }^{\circledR}$ is shown in Fig. 8. Results obtained in monocytes derived from OT patients showed that only the RNAdjuvant ${ }^{\circledR}$ induced an increased expression of CD80 compared to both negative control and other adjuvants in pre and post-chemotherapy samples. The expression of CD86 and HLA-DR was not significantly increased by adjuvants compared to the negative control. As for the OMA samples, the HLA-DR was down regulated by RNAdjuvant ${ }^{\circledR}$ in OT samples without reaching a statistical significance (Fig. 9; Additional file 1: Figure S6). Results obtained in monocytes from healthy 
$\mathrm{mDC}$ CD80

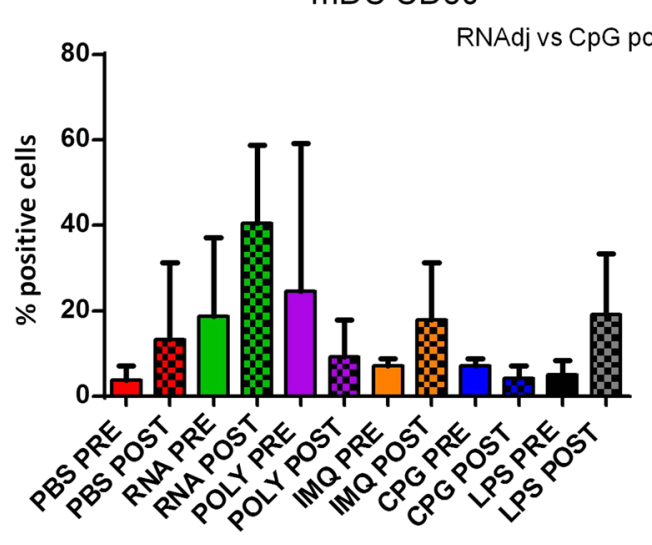

$\mathrm{mDC}$ CD86

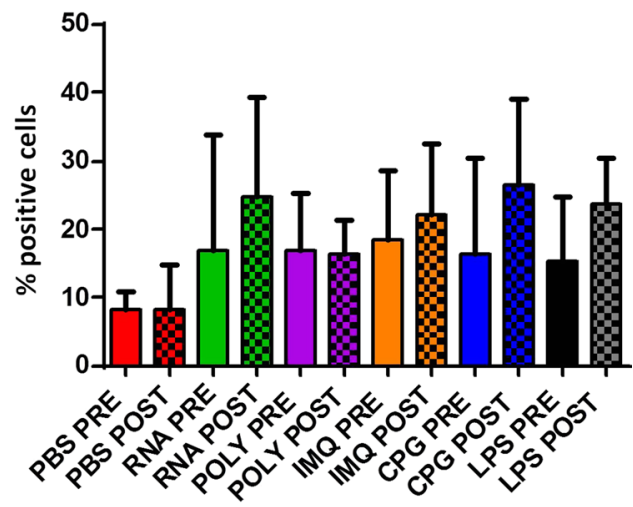

mDC HLA-DR

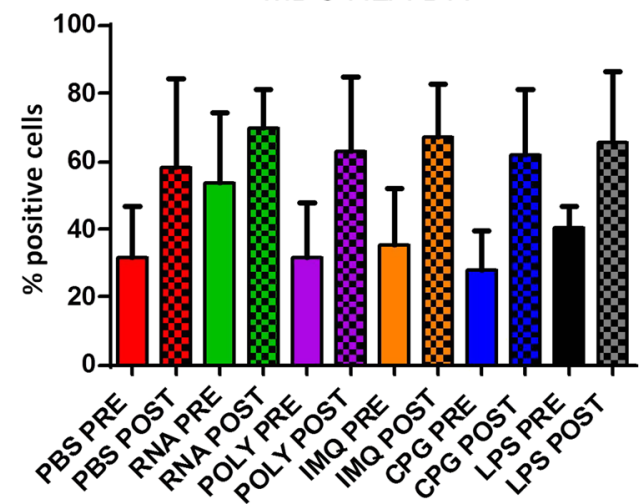

Fig. 6 Expression of markers induced by adjuvants in circulating mDCs from lung ca patients. Each of the indicated markers was evaluated by flow cytometry on cells after ex vivo treatment with adjuvants. Samples from lung cancer patients were collected pre and post-chemotherapy. Results are expressed of percentage of positive cells in the analyzed samples

subjects were similar to those obtained in pDCs and mDCs of the same subjects (Fig. 10).

\section{T cell maturation induced by the adjuvants}

The effect on $\mathrm{CD} 4^{+} \mathrm{T}$ cell phenotyping induced by APCs treated with different adjuvants was evaluated in PBMCs 7 days post-treatment ex vivo. Results showed that, at baseline, cancer patients pre and post-chemotherapy had a significantly lower $\%$ of naïve T cells (TN) compared to healthy subjects. Treatment with RNAdjuvant ${ }^{\circledR}$ induced a significant further reduction of TN in all subjects, while all other adjuvants did not have a significant effect. Concerning the effector T cells (TE), OMA patients pre and post-chemotherapy showed at baseline a significantly higher percentage of cells compare to healthy subjects. The same pattern was observed in OT patients only pre-chemotherapy, while those post-chemotherapy did not differ from the healthy subject. Treatment with adjuvants did not induce any difference compared to negative control. Central memory T cells (TCM) were slightly increased in OT patients pre and post-chemotherapy compared to healthy subjects without reaching a statistical significance; moreover, treatment with adjuvants did not induce any difference compared to negative control in samples from cancer patients. Effector memory $\mathrm{T}$ cells (TEM) were significantly increased in OMA patients pre and post-chemotherapy compared to healthy subjects; also in this case, treatment with adjuvants did not induce any difference compared to negative control in samples from cancer patients. Overall, pre and post-chemotherapy samples did not show any significant difference (Fig. 11). 

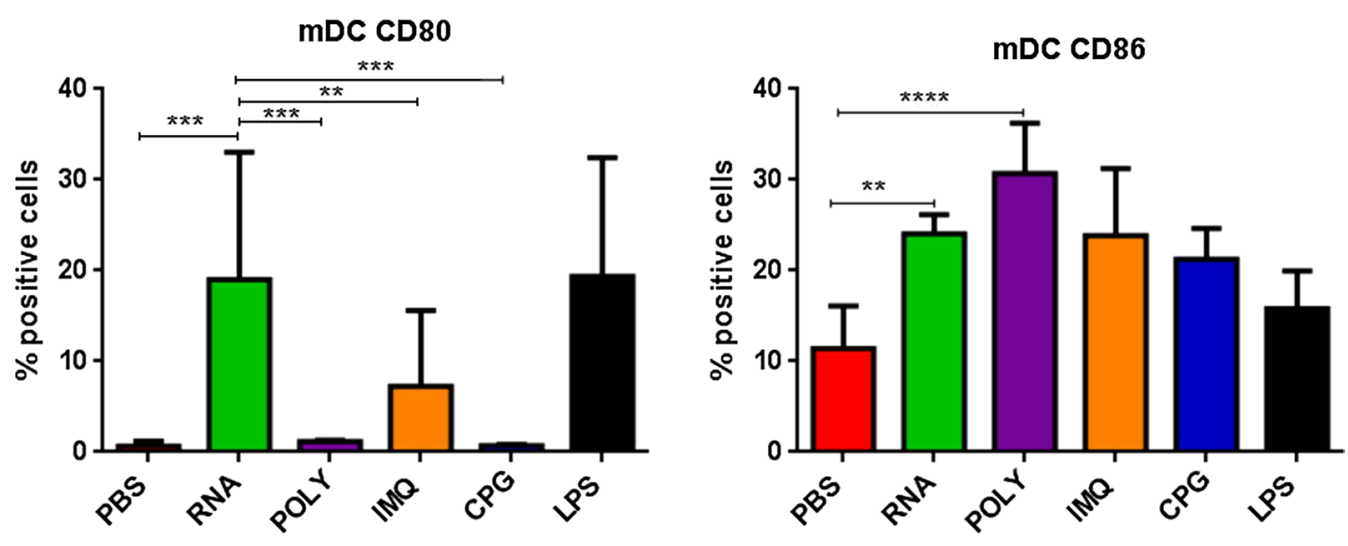

mDC DR

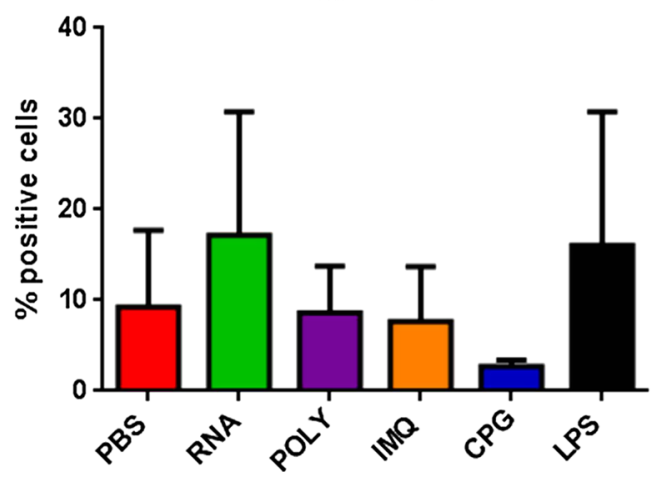

Fig. 7 Expression of markers induced by adjuvants in circulating mDCs from healthy subjects. Each of the indicated markers was evaluated by flow cytometry on cells after ex vivo treatment with adjuvants. Results are expressed of percentage of positive cells in the analyzed samples

\section{Cytokine and chemokines pattern induced in circulating APCs by different adjuvant}

The level of cytokines Th1 (IL-2 and IFNY), Th2 (IL-4), pro-inflammatory (IL-6, IL-1Beta, TNF-alpha, MIF) immunosuppressive (IL-10), chemotactic (IL-16) as well as growth factor (GM-CSF), $\alpha$-chemokines (CX3CL1, CXCL1, CXCL10, CXCL11, CXCL12, CXCL13, CXCL16, CXCL2, CXCL5, CXCL6) and $\beta$-chemokines (CCL1 CCL11, CCL13, CCL15, CCL17, CCL19, CCL2, CCL20, CCL21, CCL22, CCL23, CCL24, CCL25, CCL26, CCL27, CCL3, CCL7, CCL8, CXCL9) was assessed by Bio-Plex in supernatants of PBMCs $24 \mathrm{~h}$ after treatment with different adjuvants (Fig. 12).

The RNAdjuvant ${ }^{\circledR}$ was the only one to induce high levels of Th1 IFN- $\gamma$ in cancer patients with no significant difference in pre and post-chemotherapy samples, although at lower level compared to healthy subjects. Pro-inflammatory cytokines were induced in cancer patients mostly by RNAdjuvant ${ }^{\circledR}$ and IMQ, at levels comparable to healthy subjects, but with lower potency in post-chemotherapy samples. None of the adjuvants induced the Th2 IL-4 cytokine, with highest concentrations of $6 \mathrm{pg} / \mathrm{ml}$ induced by $\mathrm{CpG}$; moreover, only RNAdjuvant ${ }^{\circledR}$ induced a modest level of the immune-inhibitory IL-10 cytokine. Regarding $\alpha$ and $\beta$ chemokines, all the adjuvants induced their expression with different potency, although the RNAdjuvant ${ }^{\circledR}$ scored always as the most potent (Fig. 12; Additional file 1: Figures S7 and S8). In most cases, no significant difference was observed between healthy subjects and cancer patients, as well as between pre and post-chemotherapy cancer patients. Chemokines with highest expression were CXCL10, CXCL11 and CXCL13 as well as CCL20, CCL3 and CCL8. Consistently, CXCL16 and CCL24 were down-regulated by all adjuvants.

\section{Discussion}

Vaccines based on peptides show a limited efficiency in eliciting immune responses and require formulations with adjuvants to potentiate their immunogenicity. However, most of the adjuvants are developed for eliciting a Th2-type response by preventive vaccines in healthy subjects. On the contrary, therapeutic cancer vaccines aim to eliciting a Th1-type response in patients affected by 



Fig. 8 Expression of markers induced by adjuvants in circulating monocytes from colon ca patients. Each of the indicated markers was evaluated by flow cytometry on cells after ex vivo treatment with adjuvants. Samples from colon cancer patients were collected pre and post-chemotherapy. Results are expressed of percentage of positive cells in the analyzed samples. Expression of individual markers induced by RNAdjuvant ${ }^{\circledR}$ in two individual patients is shown as plot

cancer and very few adjuvants are available for this purpose. The immunological responsiveness to such adjuvants in cancer patients undergoing chemotherapy has not been previously assessed. In the present study we aimed to verify the level of responsiveness to the adjuvants mostly used in therapeutic cancer vaccine trials, comparing patients affected by colon cancer and nonsmall cell lung carcinoma, pre and post-chemotherapy, to healthy subjects.

This study was carried out using a multiparametric analysis in an ex vivo setting, treating PBMCs from lung and colon cancer patients as well as from healthy controls with RNAdjuvant ${ }^{\circledR}$, Poly I:C, IMQ and CpG. The results showed that the basal expression of the CD80, CD86 and HLA-DR molecules in monocytes, myeloid dendritic cells (mDCs) and plasmacytoid dendritic cells (pDCs) was largely comparable between healthy subjects and cancer patients, before and after the chemotherapy. However, the basal expression of CD80 and HLA-DR showed a trend of increase after the chemotherapy, possibly suggesting the positive effect of cisplatin on immune cell differentiation and antigen presentation [39-41].

The effects of the adjuvants on pDCs were very limited in cancer patients, pre and post-chemotherapy, compared to healthy subjects. Only the RNAdjuvant ${ }^{\circledR}$ induced a statistically significant increased expression of CD80 and HLA-DR in OT patients compared to the negative control, in pre and post-treatment samples respectively. The effects of adjuvants on mDCs in pre-chemotherapy samples from OMA patients showed that RNAdjuvant ${ }^{\circledR}$ and 

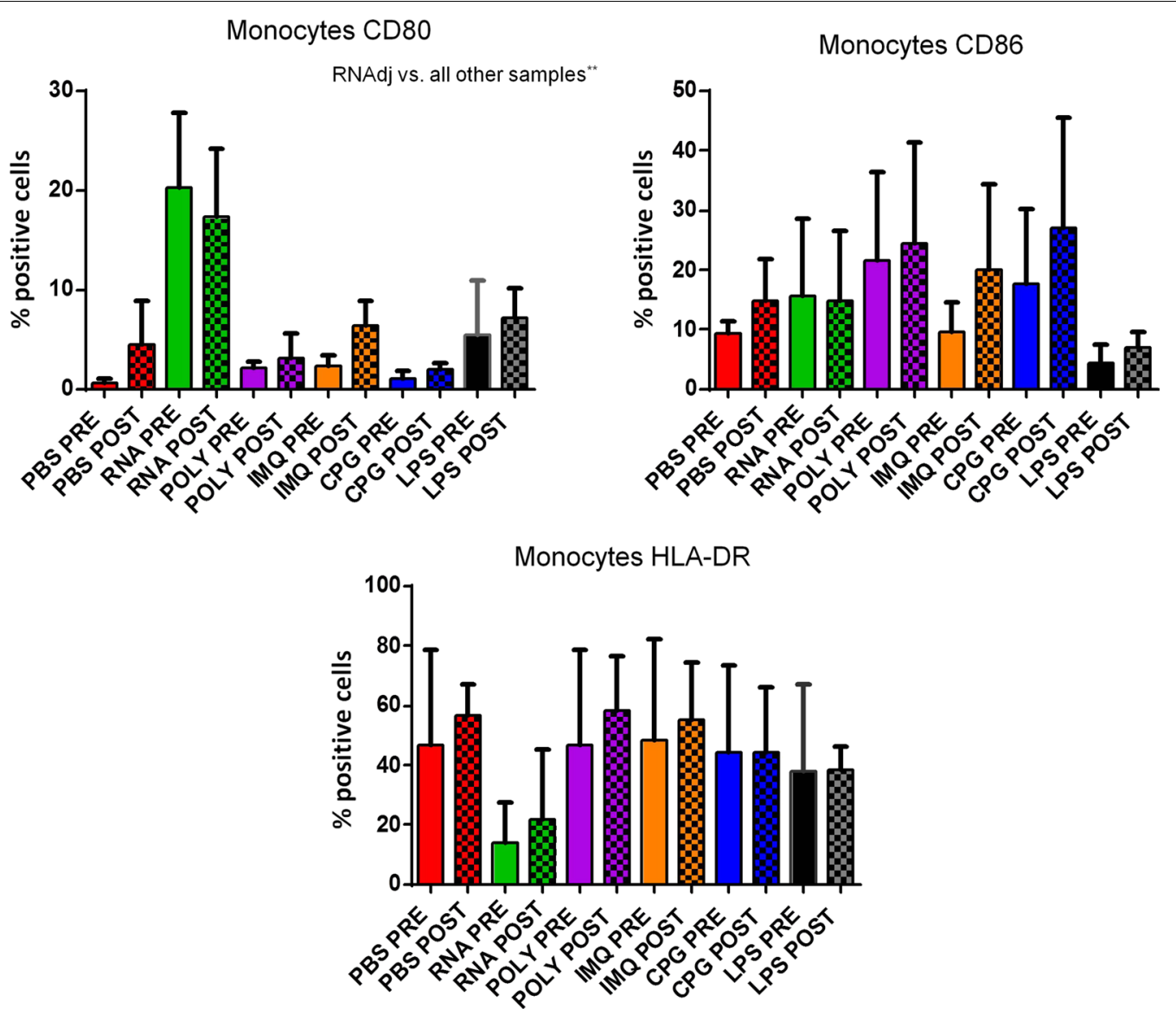

Fig. 9 Expression of markers induced by adjuvants in circulating monocytes from lung ca patients. Each of the indicated markers was evaluated by flow cytometry on cells after ex vivo treatment with adjuvants. Samples from lung cancer patients were collected pre and post-chemotherapy. Results are expressed of percentage of positive cells in the analyzed samples

CpG were able to induce a statistical significant activation of the CD80 and CD86 maturation markers compared to negative samples, respectively. On the contrary, effects on samples from OT patients were not statistically significant. The RNAdjuvant ${ }^{\circledR}$ induced the strongest activation of CD80 expression in monocytes from cancer patients, pre and post-chemotherapy, compared to negative control and other adjuvants. Poly I:C was the only adjuvant to induce an increased expression of CD86 compared to negative control in monocytes from OMA patients prechemotherapy. Overall, the results on activation markers showed that cancer patients are still responsive to adjuvants, each of them used in the present study at the individual optimal concentration. A degree of potency was observed, but the RNAdjuvant ${ }^{\circledR}$ scored as the most effective adjuvant. Interestingly, cell subtypes analyzed in the study have been shown to have different expression levels for the individual Toll Like Receptors and should be responsive only to the specific ligands. Indeed, mDCs have high expression of TLR3 as well as relative expression of TLRs7 and 8, but no expression of TLR9, and should respond to all adjuvants tested in the study with exception of $\mathrm{CpG}$ [42]. On the contrary, pDCs have high expression only of TLR7 and 9 and should respond to all adjuvants tested with exception of Poly I:C [42]. Monocytes, have low expression of TLRs7, 8 and 9 and should moderately respond to all adjuvants tested with exception of Poly I:C. However, if pDCs are present in cell culture, monocytes have been shown to respond also to the TLR9 agonist CpG [43]. According to the latter evidence, it could be predicted that in a context of mixed population (such as PBMCs) the individual subtypes may respond to TLR agonists even when the target TLRs show low expression. Data obtained after chemotherapy protocols including cisplatin or analogues suggested a positive effect on immune cell differentiation and antigen presentation, as observed in the baseline expression of the markers, although the responsiveness to adjuvants appeared to be reduced. The biological mechanisms of 

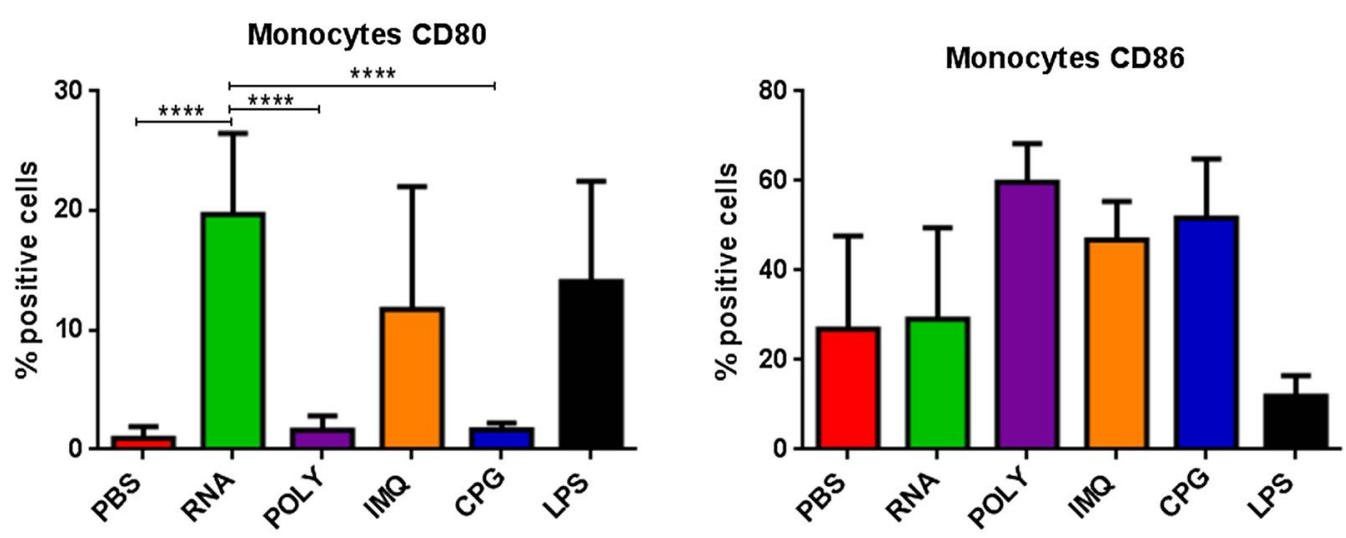

Monocytes DR

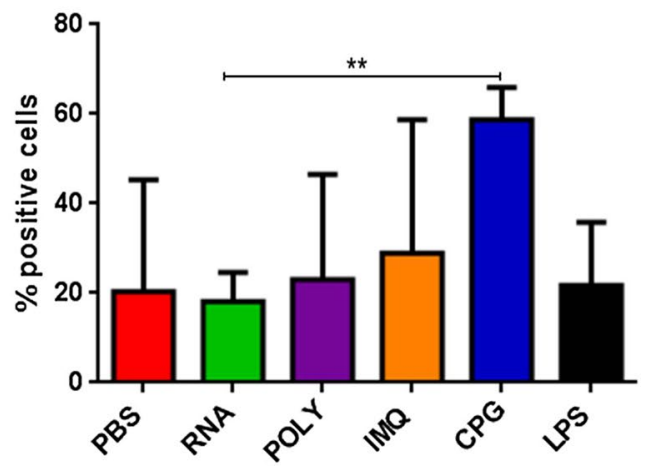

Fig. 10 Expression of markers induced by adjuvants in circulating monocytes from healthy subjects. Each of the indicated markers was evaluated by flow cytometry on cells after ex vivo treatment with adjuvants. Results are expressed of percentage of positive cells in the analyzed samples

such apparent discrepancy need to be further evaluated by increasing the number of samples analyzed.

The ability of APCs treated with adjuvants to induce activation of naïve $\mathrm{CD} 4^{+} \mathrm{T}$ cells into effector cells was assessed ex vivo. The results showed that, at baseline, pre and post-chemotherapy cancer patients have a significantly lower percentage of naïve T cells (TN) compared to healthy subjects. This would suggest that the pathological condition, regardless the chemotherapy, may have an impact on the fraction of $\mathrm{T}$ cells available to respond to new antigens. Such a reduction was further induced by treatment with RNAdjuvant ${ }^{\circledR}$, while no effect was observed upon treatment with the other adjuvants. Interestingly, colon and lung cancer patients showed a different pattern for the other phenotypes. Indeed, at baseline, while OMA samples showed a significantly higher percentage of TE and TEM compared to healthy subjects, OT patients showed a higher percentage of TCM compared to healthy subjects. For all the latter phenotypes, treatment with adjuvants did not induce a significant change in the percentage compared to the negative control. Overall, no statistical deviation from data in healthy subjects was observed in cancer patients and no difference was observed between samples from cancer patients pre and post-chemotherapy. This is of high relevance, indicating that the pool of effector and memory $\mathrm{CD} 4^{+}$ $\mathrm{T}$ cells is fully preserved in cancer patients. In particular, effector memory (TEM) and central memory (TCM) cells are capable of circulating in lymphoid (TCM) as well as non-lymphoid compartments (TEM) $[44,45]$. Therefore, upon contact with the appropriate antigen, effector memory cells can execute effector functions instantly, whereas central or lymphoid memory cells can rapidly proliferate, expanding and acquiring effector functions.

Among the adjuvants evaluated in the present study, RNAdjuvant ${ }^{\circledR}$ was the only one to induce high levels of Th1 IFN- $\gamma$ in cancer patients with no significant difference in pre and post-chemotherapy samples. On the contrary, none of the adjuvants induced IL- 4 which is considered one the major Th2 cytokines. Such results 


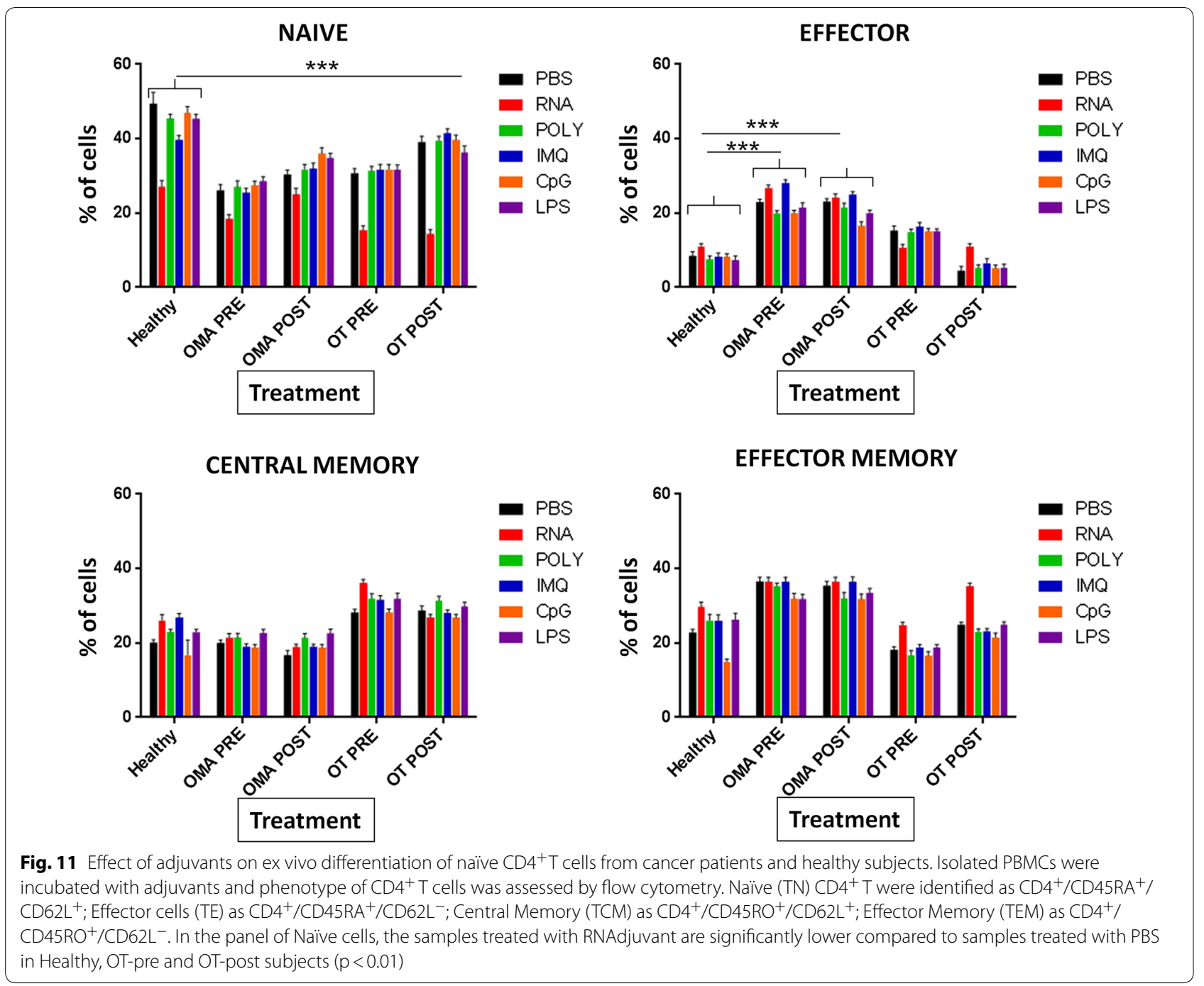

suggest that RNAdjuvant ${ }^{\circledR}$ represents the most potent Th1 inducer to be combined in a therapeutic cancer vaccine formulation. The same adjuvant is also the most potent inducer of pro-inflammatory cytokines as well as of $\alpha$ and $\beta$ chemokines, suggesting a great capability of initiating the innate immune response coupled to the ability of recruiting immune cells, especially $\mathrm{T}$ cells, for potentiating the effector arm of anti-cancer immunity. Interestingly, the biological meaning of the down-expression of CXCL16 and CCL24 induced by all adjuvants needs to be further investigated in a larger experimental setting. In most cases, although at lower level compared to healthy subjects, cytokine level in cancer patients was significantly high and no statistical difference was observed between pre and post-chemotherapy samples. In some cases, OMA patients showed a higher level of cytokine production compared to OT patients. Such an observation needs to be confirmed on a larger group of patients. 


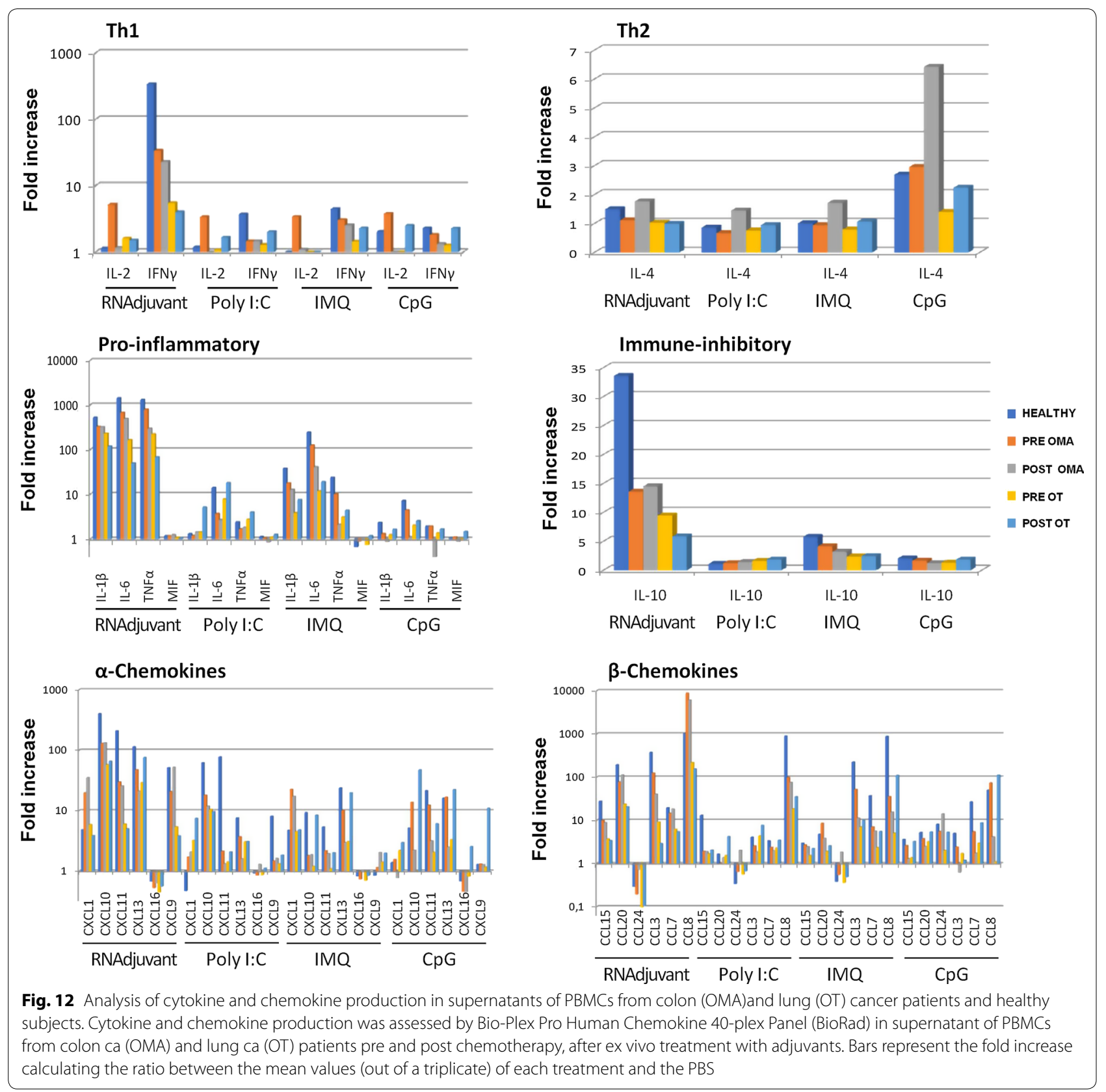

\section{Conclusion}

The immunomodulatory profile of the RNAdjuvant ${ }^{\circledR}$ appears to be potent and complete compared to the other adjuvants widely used in cancer vaccine clinical trials. Moreover, our results showed that most of the effects in cancer patients, although often of lower potency compared to healthy subjects, are suggestive of a valuable immune responsiveness. Furthermore, the limited differences observed between pre and postchemotherapy samples indicated that cancer patients may well respond to therapeutic cancer vaccines even after chemotherapy. 


\section{Supplementary information}

Supplementary information accompanies this paper at https://doi. org/10.1186/s12967-020-02218-x.

Additional file 1: Figure S1. Expression of markers induced by adjuvants in circulating pDCs from colon ca patients. Each of the indicated markers was evaluated by flow cytometry on cells after ex vivo treatment with adjuvants. Samples from colon cancer patients were collected pre and post-chemotherapy. Results are expressed of percentage of positive cells in the analyzed samples. Figure S2. Expression of markers induced by adjuvants in circulating pDCs from lung ca patients. Each of the indicated markers was evaluated by flow cytometry on cells after ex vivo treatment with adjuvants. Samples from lung cancer patients were collected pre and post-chemotherapy. Results are expressed of percentage of positive cells in the analyzed samples. Figure S3. Expression of markers induced by adjuvants in circulating $\mathrm{mDC}$ f from colon ca patients. Each of the indicated markers was evaluated by flow cytometry on cells after ex vivo treatment with adjuvants. Samples from colon cancer patients were collected pre and post-chemotherapy. Results are expressed of percentage of positive cells in the analyzed samples. Figure S4. Expression of markers induced by adjuvants in circulating mDCs from lung ca patients. Each of the indicated markers was evaluated by flow cytometry on cells after ex vivo treatment with adjuvants. Samples from lung cancer patients were collected pre and post-chemotherapy. Results are expressed of percentage of positive cells in the analyzed samples. Figure S5. Expression of markers induced by adjuvants in circulating monocytes from colon ca patients. Each of the indicated markers was evaluated by flow cytometry on cells after ex vivo treatment with adjuvants. Samples from colon cancer patients were collected pre and post-chemotherapy. Results are expressed of percentage of positive cells in the analyzed samples. Figure S6. Expression of markers induced by adjuvants in circulating monocytes from lung ca patients. Each of the indicated markers was evaluated by flow cytometry on cells after ex vivo treatment with adjuvants. Samples from lung cancer patients were collected pre and post-chemotherapy. Results are expressed of percentage of positive cells in the analyzed samples. Figure S7. Analysis of alpha chemokine production in supernatants of PBMCs from cancer patients and healthy subjects. Cytokine and chemokine production was assessed by Bio-Plex Pro Human Chemokine 40-plex Panel (BioRad) in supernatant of PBMCs treated ex vivo with adjuvants. Figure S8. Analysis of beta chemokine production in supernatants of PBMCs from cancer patients and healthy subjects. Cytokine and chemokine production was assessed by Bio-Plex Pro Human Chemokine 40-plex Panel (BioRad) in supernatant of PBMCs treated ex vivo with adjuvants.

\section{Abbreviations}

APC: antigen-presenting cell; CCL-n: chemokine (C-C motif) ligands; CTL: cytotoxic T lymphocytes; CXCL-n: chemokine (C-X-C motif) ligands; GM-CSF: Granulocyte-Macrophage Colony-Stimulating Factor; HCC: hepatocellular carcinoma; IFN- $\gamma$ : interferon gamma; IL-n: interleukins; IMQ: imiquimod; LPS: lipopolysaccharide; mDC: myeloid dendritic cell; MIF: migration inhibitory factor; OMA: colon cancer patients; OT: lung cancer patients; PAMPs: pathogenassociated molecular patterns; PBMCs: peripheral blood mononuclear cells; PBS: phosphate-buffered saline; pDC: plasmacytoid dendritic cell; Poly I:C: polyinosinic-polycytidylic acid; PRRs: pattern recognition receptors; RIG-I: retinoic acid-inducible gene 1-like receptor; TCM: central memory $T$ cell; TE: effector T cell; TEM: effector memory T cell; Th1:Thelper 1;Th2:T helper 2; TLRs: toll-like receptors; TN: Naïve T cells; TNF-alpha: tumor necrosis factor a.

\section{Acknowledgements}

We are indebted to CureVac for providing the RNAdjuvant $\left.{ }^{(}\right)$.

\section{Authors' contributions}

$A M, C M$ and BC carried out all experimental procedures. AA and MB enrolled and provided samples from colon ca patients. AM and El enrolled and provided samples from lung ca patients. AC and RR performed and analyzed cytokine analyses. MLT and FMB participated in the design of the study and analysis of data. LB and MT conceived and coordinated the study. AM and LB drafted the manuscript. All authors read and approved the final manuscript.

\section{Funding}

EU FP7 Project Cancer Vaccine development for Hepatocellular CarcinomaHEPAVAC (Grant Nr. 602893) (LB); Transcan2-HEPAMUT project (Grant Nr. 643638) (LB); Italian Ministry of Health through Institutional "Ricerca Corrente" (LB); POR FESR 2014/2020 "Campania OncoTerapie" (LB). MT and MB are funded by HEPAVAC. AM is funded by "Ricerca Corrente". CM, BC are funded by POR FESR 2014/2020 "NanoCAN".

\section{Availability of data and materials}

Data and material are available upon request.

\section{Ethics approval and consent to participate}

The study has been approved by the institutional ethical committee and all patients have signed the informed consent.

\section{Consent for publication}

The corresponding author has received consent for publication.

\section{Competing interests}

The authors declare that they have no competing interests.

\section{Author details}

${ }^{1}$ Laboratory of Cancer Immunoregulation, Istituto Nazionale per lo Studio e la Cura dei Tumori, IRCCS "Fondazione Pascale", Via Mariano Semmola, 80131 Naples, Italy. ${ }^{2}$ GI Medical Oncology, Istituto Nazionale per lo Studio e la Cura dei Tumori, IRCCS "Fondazione Pascale", Naples, Italy. ${ }^{3}$ Thoracic Medical Oncology, Istituto Nazionale per lo Studio e la Cura dei Tumori, IRCCS "Fondazione Pascale", Naples, Italy. ${ }^{4}$ Environmental, Biological and Pharmaceutical Science and Technology Dept, Università della Campania "Luigi Vanvitelli", Caserta, Italy. ${ }^{5}$ Laboratory of Molecular Biology and Viral Oncology, Istituto Nazionale per lo Studio e la Cura dei Tumori, IRCCS "Fondazione Pascale", Naples, Italy.

Received: 16 September 2019 Accepted: 10 January 2020

Published online: 23 January 2020

\section{References}

1. DeMaria PJ, Bilusic M. Cancer vaccines. Hematol Oncol Clin North Am. 2019;33:199-214

2. Circelli L, Tornesello M, Buonaguro FM, Buonaguro L. Use of adjuvants for immunotherapy. Hum Vaccin Immunother. 2017;13:1774-7.

3. Pasquale AD, Preiss S, Silva FT, Garcon N. Vaccine adjuvants: from 1920 to 2015 and beyond. Vaccines. 2015;3:320-43.

4. Moyer TJ, Zmolek AC, Irvine DJ. Beyond antigens and adjuvants: formulating future vaccines. J Clin Invest. 2016;126:799-808.

5. Bonam SR, Partidos CD, Halmuthur SKM, Muller S. An overview of novel adjuvants designed for improving vaccine efficacy. Trends Pharmacol Sci. 2017;38:771-93.

6. Tan X, Sun L, Chen J, Chen ZJ. Detection of microbial infections through innate immune sensing of nucleic acids. Annu Rev Microbiol. 2018:72:447-78.

7. Temizoz B, Kuroda E, Ishii KJ. Combination and inducible adjuvants targeting nucleic acid sensors. Curr Opin Pharmacol. 2018;41:104-13.

8. Smith M, Garcia-Martinez E, Pitter MR, Fucikova J, Spisek R, Zitvogel L, et al. Trial watch: toll-like receptor agonists in cancer immunotherapy. Oncoimmunology. 2018;7:e1526250

9. Zhu X, Nishimura F, Sasaki K, Fujita M, Dusak JE, Equchi J, et al. Toll like receptor-3 ligand poly-ICLC promotes the efficacy of peripheral vaccinations with tumor antigen-derived peptide epitopes in murine CNS tumor models. J Transl Med. 2007;5:10.

10. Martins KA, Bavari S, Salazar AM. Vaccine adjuvant uses of poly-IC and derivatives. Expert Rev Vaccines. 2015:14:447-59.

11. Kyi C, Roudko V, Sabado R, Saenger Y, Loging W, Mandeli J, et al. Therapeutic immune modulation against solid cancers with intratumoral poly-ICLC: a pilot trial. Clin Cancer Res. 2018;24:4937-48.

12. Vollmer J, Krieg AM. Immunotherapeutic applications of CpG oligodeoxynucleotide TLR9 agonists. Adv Drug Deliv Rev. 2009;61:195-204. 
13. Bode C, Zhao G, Steinhagen F, Kinjo T, Klinman DM. CpG DNA as a vaccine adjuvant. Expert Rev Vaccines. 2011;10:499-511.

14. Jackson S, Lentino J, Kopp J, Murray L, Ellison W, Rhee M, et al. Immunogenicity of a two-dose investigational hepatitis B vaccine, HBsAg-1018, using a toll-like receptor 9 agonist adjuvant compared with a licensed hepatitis B vaccine in adults. Vaccine. 2018;36:668-74.

15. Van HN, Fox CB, Granger B, Evers T, Joshi SW, Nana Gl, et al. A formulated TLR7/8 agonist is a flexible, highly potent and effective adjuvant for pandemic influenza vaccines. Sci Rep. 2017;7:46426.

16. Smith AJ, Li Y, Bazin HG, St-Jean JR, Larocque D, Evans JT, et al. Evaluation of novel synthetic TLR7/8 agonists as vaccine adjuvants. Vaccine. 2016;34:4304-12.

17. Temizoz B, Kuroda E, Ishii KJ. Vaccine adjuvants as potential cancer immunotherapeutics. Int Immunol. 2016;28:329-38.

18. Ziegler A, Soldner C, Lienenklaus S, Spanier J, Trittel S, Riese P, et al. A new RNA-based adjuvant enhances virus-specific vaccine responses by locally triggering TLR- and RLH-dependent effects. J Immunol. 2017;198:1595-605

19. Heidenreich R, Jasny E, Kowalczyk A, Lutz J, Probst J, Baumhof P, et al. A novel RNA-based adjuvant combines strong immunostimulatory capacities with a favorable safety profile. Int J Cancer. 2015;137:372-84.

20. Galluzzi L, Buque A, Kepp O, Zitvogel L, Kroemer G. Immunological effects of conventional chemotherapy and targeted anticancer agents. Cancer Cell. 2015;28:690-714

21. Bracci L, Schiavoni G, Sistigu A, Belardelli F. Immune-based mechanisms of cytotoxic chemotherapy: implications for the design of novel and rationale-based combined treatments against cancer. Cell Death Differ. 2014;21:15-25.

22. Romero Al, Chaput N, Poirier-Colame V, Rusakiewicz S, Jacquelot $N$, Chaba K, et al. Regulation of CD4(+)NKG2D(+) Th1 cells in patients with metastatic melanoma treated with sorafenib: role of IL-15Ralpha and NKG2D triggering. Cancer Res. 2014;74:68-80.

23. Vollaard A, Schreuder I, Slok-Raijmakers L, Opstelten W, Rimmelzwaan G, Gelderblom H. Influenza vaccination in adult patients with solid tumours treated with chemotherapy. Eur J Cancer. 2017;76:134-43.

24. Wumkes ML, van der Velden AM, Los M, Leys MB, Beeker A, Nijziel MR, et al. Serum antibody response to influenza virus vaccination during chemotherapy treatment in adult patients with solid tumours. Vaccine. 2013;31:6177-84.

25. Rieger CT, Liss B, Mellinghoff S, Buchheidt D, Cornely OA, Egerer G, et al. Anti-infective vaccination strategies in patients with hematologic malignancies or solid tumors-guideline of the infectious diseases working party (AGIHO) of the German Society for Hematology and Medical Oncology (DGHO). Ann Oncol. 2018;29:1354-65.

26. Dong W, Wei R, Shen H, Ni Y, Meng L, Du J. Combination of DC vaccine and conventional chemotherapeutics. Anticancer Agents Med Chem. 2016;16:558-67.

27. Buonaguro L, Tornesello ML, Tagliamonte M, Gallo RC, Wang LX, KaminLewis $\mathrm{R}$, et al. Baculovirus-derived human immunodeficiency virus type 1 virus-like particles activate dendritic cells and induce ex vivo T-cell responses. J Virol. 2006:80:9134-43.

28. Buonaguro L, Tornesello ML, Gallo RC, Marincola FM, Lewis GK, Buonaguro FM. Th2 polarization in peripheral blood mononuclear cells from human immunodeficiency virus (HIV)-infected subjects, as activated by HIV virus-like particles. J Virol. 2009;83:304-13.

29. Buonaguro L, Tornesello ML, Jewis GK, Buonaguro FM. Short communication: limited induction of IL-10 in PBMCs from HIV-infected subjects treated with HIV-VLPs. AIDS Res Hum Retrovir. 2009:25:819-22.

30. Buonaguro L, Petrizzo A, Tornesello M, Napolitano M, Martorelli D, Castello G, et al. Immune signatures in human PBMCs of idiotypic vaccine for HCV-related lymphoproliferative disorders. J TransI Med. 2010;8:18.
31. Circelli L, Petrizzo A, Tagliamonte M, Tornesello ML, Buonaguro FM, Buonaguro $L$. systems biology approach for cancer vaccine development and evaluation. Vaccines. 2015:3:544-55.

32. Petrizzo A, Tornesello ML, Napolitano M, D'Alessio G, Salomone MA, Dolcetti $R$, et al. Multiparametric analyses of human PBMCs loaded ex vivo with a candidate idiotype vaccine for HCV-related lymphoproliferative disorders. PLoS ONE. 2012;7:e44870.

33. Petrizzo A, Tagliamonte M, Tornesello ML, Buonaguro FM, Buonaguro L. Prediction of individual immune responsiveness to a candidate vaccine by a systems vaccinology approach. J Transl Med. 2014;12:11.

34. Circelli L, Petrizzo A, Tagliamonte M, Heidenreich R, Tornesello ML, Buonaguro FM, et al. Immunological effects of a novel RNA-based adjuvant in liver cancer patients. Cancer Immunol Immunother. 2017;66:103-12.

35. Miceli M, Dell'Aversana C, Russo R, Rega C, Cupelli L, Ruvo M, et al. Secretome profiling of cytokines and growth factors reveals that neuroglial differentiation is associated with the down-regulation of chemokine ligand 2 (MCP-1/CCL2) in amniotic fluid derived-mesenchymal progenitor cells. Proteomics. 2016;16:674-88.

36. Hartmann G, Weeratna RD, Ballas ZK, Payette P, Blackwell S, Suparto I, et al. Delineation of a $\mathrm{CpG}$ phosphorothioate oligodeoxynucleotide for activating primate immune responses in vitro and in vivo. J Immunol. 2000;164:1617-24.

37. Lever AR, Park H, Mulhern TJ, Jackson GR, Comolli JC, Borenstein JT, et al. Comprehensive evaluation of poly(l:C) induced inflammatory response in an airway epithelial model. Physiol Rep. 2015;3:e12334.

38. Rizzo MD, Crawford RB, Bach A, Sermet S, Amalfitano A, Kaminski NE. Imiquimod and interferon-alpha augment monocyte-mediated astrocyte secretion of MCP-1, IL-6 and IP-10 in a human co-culture system. J Neuroimmunol. 2019;333:576969.

39. Hu J, Kinn J, Zirakzadeh AA, Sherif A, Norstedt G, Wikstrom AC, et al. The effects of chemotherapeutic drugs on human monocyte-derived dendritic cell differentiation and antigen presentation. Clin Exp Immunol. 2013;172:490-9.

40. Novellino PS, Trejo YG, Beviacqua M, Bordenave RH, Rumi LS. Cisplatin containing chemotherapy influences HLA-DR expression on monocytes from cancer patients. J Exp Clin Cancer Res. 1999;18:481-4.

41. Rivas-Fuentes S, Iglesias AH, Trejo AG, Castro DYC, Figueroa NI, Perez $\mathrm{TA}$, et al. Restoration of peripheral intermediate and classical monocytes expressing HLA-DR in patients with lung adenocarcinoma after platinum-based chemotherapy. Technol Cancer Res Treat. 2018;17:1533033818764720.

42. Schreibelt G, Tel J, Sliepen KH, Itez-Ribas D, Figdor CG, Adema GJ, et al. Toll-like receptor expression and function in human dendritic cell subsets: implications for dendritic cell-based anti-cancer immunotherapy. Cancer Immunol Immunother. 2010;59:1573-82.

43. Hornung V, Rothenfusser S, Britsch S, Krug A, Jahrsdorfer B, Giese T, et al. Quantitative expression of toll-like receptor 1-10 mRNA in cellular subsets of human peripheral blood mononuclear cells and sensitivity to $\mathrm{CpG}$ oligodeoxynucleotides. J Immunol. 2002;168:4531-7.

44. Sallusto F, Lenig D, Forster R, Lipp M, Lanzavecchia A. Two subsets of memory T lymphocytes with distinct homing potentials and effector functions. Nature. 1999:401:708-12.

45. Farber DL, Yudanin NA, Restifo NP. Human memory T cells: generation, compartmentalization and homeostasis. Nat Rev Immunol. 2014;14:24-35.

\section{Publisher's Note}

Springer Nature remains neutral with regard to jurisdictional claims in published maps and institutional affiliations. 